\title{
BMJ Open Exploring the enablers and barriers to implementing the Medication Appropriateness Tool for Comorbid Health conditions during Dementia (MATCH-D) criteria in Australia: a qualitative study
}

Amy Theresa Page, ${ }^{1,2}$ Rhonda Marise Clifford, ${ }^{2}$ Kathleen Potter, ${ }^{1}$ Liza Seubert, ${ }^{2}$ Andrew J McLachlan, ${ }^{3,4}$ Xaysja Hill, ${ }^{2}$ Stephanie King, ${ }^{2}$ Vaughan Clark, ${ }^{2}$ Cristin Ryan, ${ }^{5}$ Nikesh Parekh, ${ }^{6}$ Christopher D Etherton-Beer ${ }^{1}$

To cite: Page AT, Clifford RM, Potter K, et al. Exploring the enablers and barriers to implementing the Medication Appropriateness Tool for Comorbid Health conditions during Dementia (MATCH-D) criteria in Australia: a qualitative study. BMJ Open 2017;7:e017906. doi:10.1136/ bmjopen-2017-017906

- Prepublication history and additional material are available. To view these files, please visit the journal online (http://dx.doi org/10.1136/bmjopen-2017017906).

Received 25 May 2017 Revised 4 July 2017 Accepted 19 July 2017

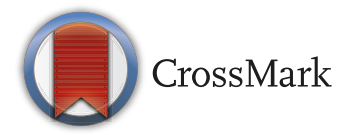

For numbered affiliations see end of article.

Correspondence to Mrs Amy Theresa Page; amy.page@uwa.edu.au

\section{ABSTRACT}

Objectives The Medication Appropriateness Tool for Comorbid Health conditions in Dementia (MATCH-D) criteria provide expert consensus guidance about medication use for people with dementia. This study aimed to identify enablers and barriers to implementing the criteria in practice.

Setting Participants came from both rural and metropolitan communities in two Australian states. Participants Focus groups were held with consumers, general practitioners, nurses and pharmacists. Outcomes: data were analysed thematically.

Results Nine focus groups were conducted. Fifty-five participants validated the content of MATCH-D, appraising them as providing patient-centred principles of care. Participants identified potential applications (including the use of MATCH-D as a discussion aid or educational tool for consumers about medicines) and suggested supporting resources.

Conclusion Participants provided insights into applying MATCH-D in practice and suggested resources to be included in an accompanying toolkit. These data provide external validation of MATCH-D and an empiric basis for their translation to practice. Following resource development, we plan to evaluate the feasibility and efficacy of implementation in practice.

\section{INTRODUCTION}

Optimising medications for older people with multiple comorbidities is difficult, ${ }^{12}$ and becomes even more complicated in people with dementia. ${ }^{3}{ }^{4}$ Effective management of multiple comorbid conditions is critical, as suboptimal management can exacerbate functional decline. ${ }^{56}$ Although older people frequently use medications to manage symptoms and to prevent disease progression and disabling events, ${ }^{78}$ there is a limited

\section{Strengths and limitations of this study}

- Participant diversity and numbers was a strength, including 55 participants from major stakeholder groups (consumers, general practitioners, nurses and pharmacists)

- Participants were recruited from rural and metropolitan regions of two different Australian states.

- The same independent facilitator ran all nine focus groups with the aid of a running sheet, which contributes to the consistency of the focus groups.

- This research has limitations common to the focus group methodology, for example, that the data are not generalisable and are limited to sentiments expressed by the participants.

understanding of appropriate pharmacological management of comorbid conditions for people living with dementia. ${ }^{3}$

The life-limiting nature of dementia may mean that the potential benefits of preventative treatments are not realised. ${ }^{9-11}$ In the context of a progressive condition such as dementia, preventative medication use for other chronic conditions such as diabetes, hypercholesterolaemia and hypertension must be balanced against adverse drug effects and the potential futility of treatment. ${ }^{12-15}$ Additionally, an individual's care goals may change as their condition progresses, meaning that optimal medication management in the early phases of dementia is not necessarily the same as in the later stages. ${ }^{16-18}$ People with dementia may be less able to reliably report symptoms both of the disease and medication side effects than their cognitively intact 
peers, which may further complicate symptom management. ${ }^{4}$ The coexistence of dementia with other chronic conditions thus poses a challenge for health professionals seeking to optimise medication use. ${ }^{4}$

We recently developed the Medication Appropriateness Tool for Comorbid Health conditions in Dementia (MATCH-D) criteria, which provide expert consensus to manage medication use for people with a diagnosis of dementia. ${ }^{19}$ Developed by a panel of 57 experts, MATCH-D include 67 consensus-based statements to guide medication use for chronic diseases in people with dementia. The statements cover the broad themes of preventative medication, symptom management, disease progression, psychoactive medications, treatment goals, principles of medication use, side effects and medication reviews. Treatment suggestions are tailored to the stage of dementia as defined by functional capacity. ${ }^{19}$

We designed the current study to support the translation of MATCH-D into clinical practice. The aim of this study was to determine stakeholders' perceptions of the enablers and barriers to the effective use of MATCH-D criteria in practice.

This study explored the enablers and barriers to the MATCH-D, expert consensus to manage medication use for people with a diagnosis of dementia.

\section{METHODS}

Ethics approval was obtained from the UWA Human Research and Ethics Committee (HREC RA/4/1/8202).

This study applied a focus group methodology to investigate the study aims and is reported in line with the Consolidated Criteria for Reporting Qualitative Research (COREQ) checklist (see online supplementary appendix 1). ${ }^{20}$

\section{Facilitator}

An independent experienced researcher (LS) facilitated focus group discussions using a topic guide (see online supplementary appendix 2). The same independent facilitator led each session to ensure uniformity in the method.

\section{Study sample and recruitment}

Each focus group consisted of participants from one of four stakeholder groups: consumers (people with dementia and carers for people with dementia), general practitioners (GPs), registered nurses and pharmacists.

Snowball sampling techniques were applied. ${ }^{21}$ Initial recruitment was via professional organisations and both professional and patient networks. These consumer support and professional representative organisations included Alzheimer's Australia WA, Pharmaceutical Society of Australia (WA), Carers WA, Consumer Health Forum of Australia and Council on the Ageing. Awareness of the research and recruitment was raised using posters, advertisements, telephone, email and social media. The authors contacted individual pharmacies, medical practices, hospitals, community nursing services, retirement villages, residential aged care facilities and professional contacts by telephone and email.

\section{Setting}

Focus groups were held in a variety of settings to suit participants' preferences, including the University of Western Australia, the workplace (GP practices) and the home environment, in September 2016. Participants came from rural, inner city and outer metropolitan locations in two Australian states (Western Australia and Victoria).

\section{Data collection}

Focus groups were conducted until data saturation occurred across the groups. Data collected at the focus group sessions comprised audio recordings, participant demographics, and researcher observations and field notes. Two or three observing researchers (AP, SK, VC, $\mathrm{XH}$ ) attended the focus groups to take detailed typed notes of each session since observations of mood, energy, enthusiasm and expression cannot be determined via transcripts alone. These detailed notes supplemented a primary verbatim transcription of the audio recording post-focus group. The collected data were collated for analysis.

\section{Data analysis}

Two researchers (AP with one of SK, XH, VC) independently hand-coded line by line all the focus group transcripts using the content analysis method to identify key themes. ${ }^{21}{ }^{22}$ The researchers compared their individual findings from the GP stakeholder focus group transcripts to reach consensus on the themes. They developed a framework of themes that was then applied to the remainder of the transcripts. This framework was modified and adapted as necessary to encompass the emergent themes from all focus groups. The major and minor themes were then discussed to consolidate and simplify the themes. Similarities and differences across the four stakeholder groups were examined and compared.

\section{RESULTS}

A total of 55 people participated in nine focus groups (table 1). Participants included 41 health professionals (22 GPs, nurses, and 10 pharmacists) and 14 consumers (2 people with dementia and 12 carers).

Three categories of themes emerged: validation, potential applications and possible resources. Participants validated the content of MATCH-D, feeling it provided guiding principles of care while remaining patient-centred. Potential applications for its use were identified, and resources were suggested to support implementation.

Within each theme, the findings are presented from the consumers, general practitioners, nurses and pharmacists. The term health professional is used where 
Table 1 Participant demographics

\begin{tabular}{|c|c|c|c|c|}
\hline \multicolumn{5}{|l|}{ Health professionals } \\
\hline & \multicolumn{2}{|c|}{ General practitioner, $\mathrm{n}=\mathbf{2 2}$} & Registered nurse, $\mathrm{n}=9$ & Pharmacists, $\mathrm{n}=10$ \\
\hline $\begin{array}{l}\text { Time as a qualified health } \\
\text { professional (years) }\end{array}$ & \multicolumn{2}{|l|}{$18 \pm 14$ years } & $18 \pm 15$ years & $18 \pm 11$ years \\
\hline $\begin{array}{l}\text { Clinical time caring for people with } \\
\text { dementia (estimated percentage) }\end{array}$ & \multicolumn{2}{|l|}{$9 \pm 5$} & $37 \pm 34$ & $11 \pm 13$ \\
\hline Practice setting & \multicolumn{2}{|c|}{$\begin{array}{l}\text { General practice, } n=20 \\
\text { Hospital, } n=2\end{array}$} & $\begin{array}{l}\text { Aged care, } n=5 \\
\text { Palliative care, } n=1 \\
\text { Hospital, } n=3\end{array}$ & $\begin{array}{l}\text { Hospital, } n=4 \\
\text { Community, } n=6 \\
\text { Medication reviews, } n=2 \\
\text { Government, } n=1 \\
\text { Academic, } n=2\end{array}$ \\
\hline \multicolumn{5}{|l|}{ Consumers } \\
\hline & & \multicolumn{2}{|c|}{$\begin{array}{l}\text { Carers for people with dementia } \\
(n=12)\end{array}$} & $\begin{array}{l}\text { People with dementia } \\
(n=2)\end{array}$ \\
\hline \multicolumn{2}{|c|}{ Years since diagnosis of person with dementia } & \multicolumn{2}{|l|}{$6 \pm 6$} & $1 \pm 1$ \\
\hline \multicolumn{2}{|c|}{ Number of days each week spent providing care } & \multicolumn{2}{|c|}{$4 \pm 3$} & $\mathrm{n} / \mathrm{a}$ \\
\hline \multicolumn{2}{|c|}{ People providing care for } & \multicolumn{2}{|c|}{$\begin{array}{l}\text { One person, } n=10 \\
\text { Two people, } n=2 \\
\text { Spouse, } n=3 \\
\text { Parent, } n=10\end{array}$} & $\mathrm{n} / \mathrm{a}$ \\
\hline
\end{tabular}

Data are presented as mean \pm SD unless otherwise specified.

Some participants worked in more than one setting, so numbers may not add up to total.

GPs, nurses and pharmacists collectively discussed the same issues; otherwise, they are referred to by individual profession.

\section{Validation}

Support to optimise medication use is needed

Participants validated the content and overall direction of MATCH-D. Consumers and health professionals alike discussed the challenge of medication management for people with dementia, and agreed that written support to optimise medication use could be beneficial.

I think that there's a very great need for it and I think this is a particularly good document. ... it's sensible, it's a sensible policy.' [GP 17]

I think that the medications to make them comfortable are an absolute must. [Consumer 5]

Address the needs of patients at different stages of dementia Participants discussed the nuances of managing comorbidities, with an emphasis on the complexities involved in providing care for people with dementia at different stages. GPs discussed the complexities and potential complications of different scenarios.

I think that it should be addressed, consider not treating infections, pneumonia is the old man's friend. You've got to go of something. It may be a better way to go than a heart attack or a sudden event or pulmonary embolus, because you're going to stop their warfarin. [GP 10]

...the treatments why bother if it's not being (?) working' [Pharmacist 5]

Guiding principles but care should remain patient-centred

Participants perceived that it was useful to have consensus statements to detail the principles of care, but emphasised that having the flexibility to tailor care to the individual was key.

...it's almost not hard and fast. It's think about reducing non-symptom control medications ...You do it on an individual basis. [GP 16]

So it'll be helpful this thing, but still it's kind of generalised you know, like a sensible way of doing things. But again to treat a patient, depends on a lot of other circumstances and the individualisation will be the most important thing... [GP 18]

\section{Potential applications}

Consumers and health professionals identified multiple potential applications for MATCH-D in practice.

MATCH-D could work as a discussion aid

Consumers and health professionals alike considered that MATCH-D could be used as a discussion aid to prompt conversations about care options. Consumers felt 
it could help suggest questions to ask both professionals and loved ones.

It would give you questions to consider to put in place ... It's a great start to having a discussion about what we should be doing. [Consumer 7]

I wonder if it could be used as a family discussion point. [Consumer 8]

Health professionals varied in their opinions about discussing treatment options with consumers from positive to apprehensive.

So you can have a risk benefit conversation ... let's have an informed discussion around what we're going to do with it. [Pharmacist 6]

It's a hard one because you don't want-people with early stage dementia are well enough to realise how... the end is and you don't want to sort of rub it in their face in a way. [GP 14]

\section{Help consumers plan for the future}

Consumers were keen to have the wishes of people living with dementia documented. They voiced an enthusiasm for advanced care planning to be a routine part of healthcare so the affected person's wishes could be respected even as their condition deteriorated. Consumers perceived the MATCH-D as a useful tool to prompt and facilitate these discussions.

And that's why a tool would be good, if there was this tool and it was just like one thing that doctors went through if it was just normal like the assessment thing then. [Consumer 1]

....all the modern medicines that we've got now are keeping us all alive longer and there's going to be a whole heap more dementia. And do we really want to live through the last stages of dementia? I would rather be taken out by a heart attack or something else before I get to the actual end stages of dementia. and slowing down the medications earlier on so that something else can take me rather than me lying there and not being able to eat and starving to death. [Consumer 5]

When the doctor realises that someone is getting dementia, to ask them directly what they would like later on in their lives. [Consumer 7]

\section{Support health professionals make difficult decisions about medication}

Health professionals expressed nervousness about making medication decisions for people with dementia, although perceived that MATCH-D could be useful to support decisions to change medication management strategies as the condition progressed.

If there was a back-up, that there was evidence that that was the appropriate thing to do, then I think there would be a greater confidence in withdrawing medication at certain points in someone's life. [GP 5]
The consumers expressed enthusiasm for health professionals to initiate conversations about treatment decisions. Consumers perceived that MATCH-D could assist health professionals to help people with dementia.

...I know it's the ideal world type thing, but at least it takes the burden off the carer ... so if there's a formal thing and a professional handles it, it opens up that world of possibility ... [Consumer 1]

Used as an educational tool for both consumers and professionals Health professionals discussed the potential for MATCH-D to be used as an educational resource for consumers.

Explain the rationale behind why you might want to stop medications. [GP 3]

Health professionals also noted that MATCH-D could be used as an educational aid for other health professionals.

I can see that it would be a very important tool for training nursing home staff and also for HMRs (Home Medicine Reviews)... it's a powerful tool because often (nurses are) the ones often providing advice on what should happen with the medications (to consumers). [Pharmacist 10]

\section{Possible resources}

Participants suggested resources that could inform a toolkit to support use in practice and suggested changes to the current format of MATCH-D. Health professionals stressed the importance of creating a resource that was easy to use.

\section{A consumer version of the criteria is needed}

Consumers and health professionals cited the importance of providing a consumer version.

So long as there's (sic) tools for families as well then I think that this is just the beginning of something much more wonderful. [Consumer 1]

'... a little brochure that goes along with it that you can give out to people that says these are the types of things your doctor will be considering or your family members doctor will be considering, based on evidence....Direct it at the family member or the actual person with dementia. [GP 7]

\section{Different, and varied, formats are required}

Participants requested that MATCH-D be available in multiple formats. GPs requested an abbreviated version for use during consultations and a referenced version of the statements with augmented detail. Pharmacists also requested a referenced version of the statements. Participants suggested additional formats, namely a checklist and an online version, as useful to support its use in practice. 
If it was online, you could have drop-downs, couldn't you? So you could have the early-, mid-stage, latestage, click on the one you want and have a dropdown with extra detail. You could even have a further drop-down with more detail, if you needed more. [GP 8]

... It's these two blue pages that are so important ... when you need the guideline you go straight to those pages. [GP 12]

...the one-page summary. But I think that a thicker document for late night reading...(sic) [GP13]

\section{Training might be needed}

Health professionals suggested that training would enable them to implement MATCH-D.

Workshops, in particular hands-on workshops like giving examples, case studies, multiple examples, it makes things easier. [GP 4]

Not all stakeholders agreed. Other GPs suggested that familiarity would come with use.

Once you've read it I think it'll stick in your head really...I think that once you've made, you've read it, it'll probably be there in your brain. [GP 16]

\section{DISCUSSION}

This research explored stakeholders' opinions on using MATCH-D in clinical practice. Participants validated the content, supporting the need for a tool such as MATCH-D to support medication management for people with dementia which could also be adapted to the individual. Participants identified multiple potential applications for its use and suggested resources to support use in practice.

Consumers and health professionals alike suggested a consumer version would be useful. A consumer version would need to use accessible language as about half of Australian adults have insufficient literacy levels to independently complete daily tasks. ${ }^{23}$ Therefore, consumer resources should be developed assuming low levels of both literacy and health literacy, although based on principles of shared decision making. ${ }^{24}{ }^{25}$ A consumer version could include paraphrased statements that communicate the MATCH-D statements using accessible language. The consumer version could explain that 'the doctor will think about ...' and to prompt consumers to 'ask about ...' or 'consider' particular topics.

Stakeholders suggested multiple formats of MATCH-D would be useful to enhance practical application. Participants expressed a desire to have access to MATCH-D in both paper and online formats, as both a checklist and abbreviated version. These suggested resources are feasible to develop and could form a toolkit for health professionals to use to support the use of the criteria in practice. As a result, we have commenced work on an online version (match-d.com.au). The process to develop a checklist will involve identifying which statements the health professional can assess as a binary yes/no outcome.

The GPs and pharmacists requested supporting references be incorporated into MATCH-D. The request for a referenced version and more detail to access the literature and evidence to support the statements within MATCH-D. However, the limited evidence base was the main reason that a consensus methodology was applied to develop the MATCH-D. ${ }^{26} 27$ This paucity of research was demonstrated in our recent systematic review on the topic found only one randomised study that investigated medication use to manage comorbidities. ${ }^{27}$ This limited evidence base means it would not be feasible to reference the MATCH-D.

This study has several strengths and limitations that are worth noting. Limitations of this research include those that are common to focus group methodology, for example, that the data are not generalisable and are limited to sentiments expressed by the participants. We mitigated against the possibility for misinterpretation by having at least two researchers independently undertake the thematic analysis and reach agreement.

The key strength of this study was the ability to recruit many participants across the stakeholder groups: participants came from both rural and metropolitan areas in two Australian states, Victoria and Western Australia. Furthermore, variation within each stakeholder group provided a diverse range of perspectives and experiences. For example, consumer groups comprised carers and people with dementia. Focus groups were conducted across a range of settings. As the facilitator has the ability to introduce bias and lead participant comments, the data quality generated from focus groups is contingent on the skill and impartiality of the facilitator. ${ }^{28}{ }^{29}$ The use of a single independent facilitator to facilitate each focus group session with the aid of a running sheet increased consistency between the groups.

Further research into the MATCH-D is needed. Once the resources to accompany the MATCH-D have been developed and evaluated, translational research should be undertaken to assess the MATCH-D for clinical application. The next steps are to undertake feasibility and pilot studies to test its application in clinical practice. After this work has been undertaken, larger studies are needed to establish whether medication management optimised using the MATCH-D resulted in improved health outcomes or quality of life.

The findings of this study provided valuable insight into the perceived use of MATCH-D in clinical practice by validating the criteria, identifying potential applications and exploring resources that could support these applications. This feedback and input will inform the implementation of MATCH-D. Once the resources outlined have been developed, the next step is to undertake research to evaluate the effects of applying MATCH-D in clinical practice.

\section{Author affiliations}

${ }^{1}$ Western Australian Centre for Health and Ageing, School of Medicine, University of Western Australia, Perth, Australia 
${ }^{2}$ School of Allied Health, University of Western Australia, Perth, Australia

${ }^{3}$ Faculty of Pharmacy and Centre for Education and Research on Ageing, University of Sydney, Sydney, New South Wales, Australia

${ }^{4}$ Concord Hospital, Sydney, New South Wales, Australia

${ }^{5}$ School of Pharmacy, Royal College of Surgeons in Ireland, Dublin, Ireland

${ }^{6}$ Brighton and Sussex Medical School, Brighton, UK

Acknowledgements The authors gratefully acknowledge the consumers, nurses, general practitioners and pharmacists who participated in these focus groups. They thank the pharmacists and Master of Pharmacy students at the University of Western Australia who participated in their pilot focus group. This work was supported by the Dementia Collaborative Research Centers and the University of Western Australia in providing a University Postgraduate Award to AP.

Contributors AP, CE-B, KP, RC, AM and CR designed the study. AP acted as chief investigator and drafted the funding application and Human Research Ethics Committee application. AP undertook recruitment along with the three Master of Pharmacy students (VC, XH, SK). AP drafted the protocol and the topic guide for the focus groups. LS moderated the focus groups as an independent facilitator. $\mathrm{VC}, \mathrm{XH}$ and SK transcribed the focus groups. AP, VC, XH and SK undertook the qualitative analysis. AP drafted the manuscript for the published study. CE-B, RC, KP, AM, LS, CR, NP provided critical review and assistance with revisions.

Funding AP was funded by a University Postgraduate Award from the University of Western Australia. The Dementia Collaborative Research Centre supported this research through a competitive grant.

Competing interests None declared.

Patient consent Obtained.

Ethics approval University of Western Australia.

Provenance and peer review Not commissioned; externally peer reviewed.

Data sharing statement Data collected and analysed for this research are available by emailing the corresponding author Amy Page, amy.page@uwa.edu.au.

Open Access This is an Open Access article distributed in accordance with the Creative Commons Attribution Non Commercial (CC BY-NC 4.0) license, which permits others to distribute, remix, adapt, build upon this work non-commercially, and license their derivative works on different terms, provided the original work is properly cited and the use is non-commercial. See: http://creativecommons.org/ licenses/by-nc/4.0/

(C) Article author(s) (or their employer(s) unless otherwise stated in the text of the article) 2017. All rights reserved. No commercial use is permitted unless otherwise expressly granted.

\section{REFERENCES}

1. O'Mahony D, O'Sullivan D, Byrne S, et al. STOPP/START criteria for potentially inappropriate prescribing in older people: version 2. Age Ageing 2015;44:213-8.

2. By the American Geriatrics Society 2015 Beers Criteria Update Expert Panel. American Geriatrics Society 2015 Updated Beers Criteria for Potentially Inappropriate Medication Use in Older Adults. J Am Geriatr Soc 2015;63:2227-46.

3. Reeve E, Bell JS, Hilmer SN. Barriers to Optimising Prescribing and Deprescribing in Older adults with dementia: a narrative review. Curr Clin Pharmacol 2015;10:168-77.

4. Bunn F, Burn AM, Goodman C, et al. Comorbidity and dementia: a scoping review of the literature. BMC Med 2014;12:192.

5. Melis RJ, Marengoni A, Rizzuto D, et al. The influence of multimorbidity on clinical progression of dementia in a populationbased cohort. PLoS One 2013;8:e84014.
6. Pasqualetti G, Tognini S, Calsolaro V, et al. Potential drug-drug interactions in alzheimer patients with behavioral symptoms. Clin Interv Aging 2015;10:1457-66.

7. Scott IA, Hilmer SN, Reeve E, et al. Reducing inappropriate polypharmacy: the process of deprescribing. JAMA Intern Med 2015;175:827-34.

8. Lee SJ, Leipzig RM, Walter LC. Incorporating lag time to benefit into prevention decisions for older adults. JAMA 2013;310:2609-10.

9. Larson EB, Shadlen MF, Wang L, et al. Survival after initial diagnosis of Alzheimer disease. Ann Intern Med 2004;140:501.

10. Wolfson $\mathrm{C}$, Wolfson DB, Asgharian M, et al. A reevaluation of the Duration of Survival after the onset of Dementia. N Engl J Med Overseas Ed 2001;344:1111-6.

11. Holmes HM, Min LC, Yee M, et al. Rationalizing prescribing for older patients with multimorbidity: considering time to benefit. Drugs Aging 2013;30:655-66.

12. Holmes HM. Rational prescribing for patients with a reduced life expectancy. Clin Pharmacol Ther 2009;85:103-7.

13. Holmes HM, Sachs GA, Shega JW, et al. Integrating palliative medicine into the care of persons with advanced dementia: identifying appropriate medication use. J Am Geriatr Soc 2008;56:1306-11.

14. Somers M, Rose E, Simmonds D, et al. Quality use of medicines in residential aged care. Aust Fam Physician 2010;39:413-6.

15. Parekh N, Page A, Ali K, et al. A practical approach to the pharmacological management of hypertension in older people. Ther Adv Drug Saf 2017;8:117-32.

16. Taghizadeh Larsson A, Österholm JH. How are decisions on care services for people with dementia made and experienced? A systematic review and qualitative synthesis of recent empirical findings. Int Psychogeriatr 2014;26:1849-62.

17. Parsons $\mathrm{C}$, Hughes CM, Passmore AP, et al. Withholding, discontinuing and withdrawing medications in dementia patients at the end of life: a neglected problem in the disadvantaged dying? Drugs Aging 2010;27:435-49.

18. Parsons C, McCann L, Passmore P, et al. Development and application of medication appropriateness indicators for persons with advanced dementia: a feasibility study. Drugs Aging 2015;32:67-77.

19. Page AT, Potter K, Clifford R, et al. Medication appropriateness tool for co-morbid health conditions in dementia: consensus recommendations from a multidisciplinary expert panel. Intern Med J 2016;46:1189-97.

20. Tong A, Sainsbury P, Craig J. Consolidated criteria for reporting qualitative research (COREQ): a 32-item checklist for interviews and focus groups. Int J Qual Health Care 2007;19:349-57.

21. Huston SA, Hobson EH. Using focus groups to inform pharmacy research. Res Social Adm Pharm 2008;4:186-205.

22. Hsieh HF, Shannon SE. Three approaches to qualitative content analysis. Qual Health Res 2005:15:1277-88.

23. Australian Bureau of Statistics. Adult Literacy, Australian Social Trends. 2008. http://www.abs.gov.au/AUSSTATS/abs@.nsf/Lookup/ 4102.0Chapter6102008

24. Baker DW. The meaning and the measure of health literacy. J Gen Intern Med 2006;21:878-83.

25. Jansen J, Naganathan V, Carter SM, et al. Too much medicine in older people? deprescribing through shared decision making. BMJ 2016;353:i2893.

26. Page A, Potter K, Clifford R, et al. Prescribing for Australians living with dementia: study protocol using the Delphi technique. BMJ Open 2015;5:e008048.

27. Page A, Etherton-Beer C, Seubert L, et al. Medication use to manage comorbidities for people with dementia: a systematic review. J Pharm Pract Res 2017.

28. Liamputtong P. Research methods in Health: foundations for evidence-based practice. 2nd edition: Oxford University Press, 2014.

29. Krueger RA, Casey MA. Focus groups: a practical guide for applied research. Fifth Edition: SAGE publications, 2014. 